MIDPI

MOL2NET, International Conference Series on Multidisciplinary Sciences

sciforum,

\title{
Hyperheuristics for indirect elicitation of outranking model's parameters in Project Portfolio Optimization
}

\author{
Nelson Rangel-Valdez ${ }^{b^{*}}$,Eduardo Fernandez ${ }^{a}$, Laura Cruz-Reyes ${ }^{b}$, Claudia Gomez- \\ Santillán ${ }^{b}$,Lucila Morales-Rodríguez ${ }^{b}$
}

${ }^{a}$ Faculty of Civil Engineering, Autonomous University of Sinaloa Culiacan, Culiacan, México. E-mail: eddyf171051@gmail.com

${ }^{b}$ Postgraduate and Research Division, National Mexican Institute of Technology/Madero Institute of Technology, Cd. Madero,

México. E-mails: [nelson.rangel, lauracruzreyes, claudia.gomez, lucila.morales\}@itcm.edu.mx

* This author is a CONACyT Research Fellow at TecNM/ITCM from Catedras CONACyt

\begin{tabular}{|l|l|}
\hline One of the main problems that face Multi-Objective Evolutionary \\
Algorithms (MOEAs) when approximating the best compromise \\
solutions is a proper a priori incorporation of the Decision Maker's \\
(DM) preferences. Particularly, when these methods rely on outranking \\
approaches, they need eliciting several parameters. Given that his task is \\
of great cognitive effort for a DM, it is performed indirectly through a \\
battery of examples that (s)he provides previously and that reflex the \\
desired preferences. So far, only metaheuristics have been used to \\
transform such examples into parameters'values of specific preference \\
models. The present research propose an architecture for a \\
hyperheuristic that integrates characterization and performance analysis \\
into the elicitation process. It is expected that a good combination the \\
metaheuristic could improve the quality of parameters estimated. \\
Keywords: Parameter Elicitation; Preference Disaggregation Analysis; \\
MOEAs; Hyperheuristic.
\end{tabular}

\section{Introduction}

Multi-Objective Evolutionary Algorithms (MOEAs) are methods which are strongly recommended to approximate the Pareto frontier whenever the characteristics of objective functions and constraints make it difficult for mathematical programming (cf. Coello, 1999). These approaches construct a solution set formed by non-dominated solutions (Coello, 2000; Coello, 2017).

Alternatively, an MOEA can approximate toward the set of best compromise solutions, i.e. the Region of Interest or RoI, through the incorporation of information about the preference of a decision maker (DM), see (Zhu and Luo, 2016). According to (Branke et al., 2016), the DM preference information 
within an MOEA search process can be motivated by the necessity of sampling of the Pareto frontier, or the reduction of the DM's cognitive effort to handle only the RoI, or because the DM's preference information reinforces the necessary selective pressure. Let us observe that the incorporation of preferences is a method that offers support to the limited capacity of the human mind to handle several conflicting objectives at the same time (Miller, 1956); so, this method has become a very powerful tool that aids in the solution of many-objective problems.

However, most of the time the behavior of a complex system often depends on parameters whose values are unknown in advance (Ling et al., 2010). Such is the case of MOEAs based on outranking approaches (Fernandez et al., 2011). The outranking approaches are methods that construct outranking relations among potential actions or decision alternatives and exploit such relations to find solutions to decision problems. These approaches have found application in approximating the RoI in Multiobjective Optimization Problems (MOPs) because they allow computational models of preferences of DM's that can be used to guide the search in MOEAs toward solutions that are closely related to his/her interests (Bechikh, 2013).

Ideally, the parameter values of an outranking approach should be defined by the DM; however, given the cognitive effort required from the DM, this task can be extremely difficult and time-consuming, and hence prohibited to be handled directly (Dias and Mousseau, 2006). This situation is aggravated in cases when a DM is not capable of providing a clear explanation of his/her decisions. These situations prevent from the use of a direct assessment of the parameter values required by a complex system. Instead, the most convenient strategy to overcome such problems lies in the use of preference disaggregation methods.

A preference disaggregation method (PDM) is an indirect elicitation approach that can indirectly infer the values of a predefined set of parameters from a set of examples provided by the DM. In these approaches, the provision of preference information is far simpler for a DM because it can be done based on decisions taken in the past or formulated recently by means of manageable examples. So far, PDMs have been implemented using evolutionary metaheuristics (Rangel-Valdez et al., 2015; CruzReyes et al., 2017), and they have shown their effectiveness on the parameter elicitation for outranking approaches used in the solution of the Portfolio Selection Problem (PSP) with the methods ELECTRE as preference models (cf. Roy, 1991). However, the success in the parameter elicitation does not depend only on the quality of the provided set examples but also on the performance of the used algorithms; in this aspect, it has been observed in other problems that the achievement of good solutions in a wider range of instances might require the use of several metaheuristics combined. Recently, hyperheuristics gain more attention because of their capacity to integrate characterization models of problem instances with a set of metaheuristics in order to improve the construction of solution in an optimization problem (Burke et al., 2009; Burke et al., 2010; Soubeiga, 2003). Based on the fact that parameter elicitation has been modeled previously by optimization problems, this work proposes the study of the impact on the parameter elicitation of outranking approaches for PSP due to the implementation of a hyperheuristic that selects adequately the best metaheuristics given the instance of the problem and the state of the search process. For this purpose, the research presents as a result an architecture that guides on how to integrate metaheurisics and performance metrics as elements of an hyperheuristic for elicitation of parameters. 


\section{Results and Discussion}

Figure 1 presents the basic architecture to create the hyperheuristc for parameter elicitation. There are four main components in the architecture: a) the definition of the reference set; $b$ ) the generation of the initial solution $P_{0} ;$ c) the performance model $\operatorname{Cost}\left(P_{a c t}\right)$ that evaluates the quality of the actual population $P_{a c t}$; and, d) the metaheuristics to improve the actual population of solutions $P_{a c t}$.

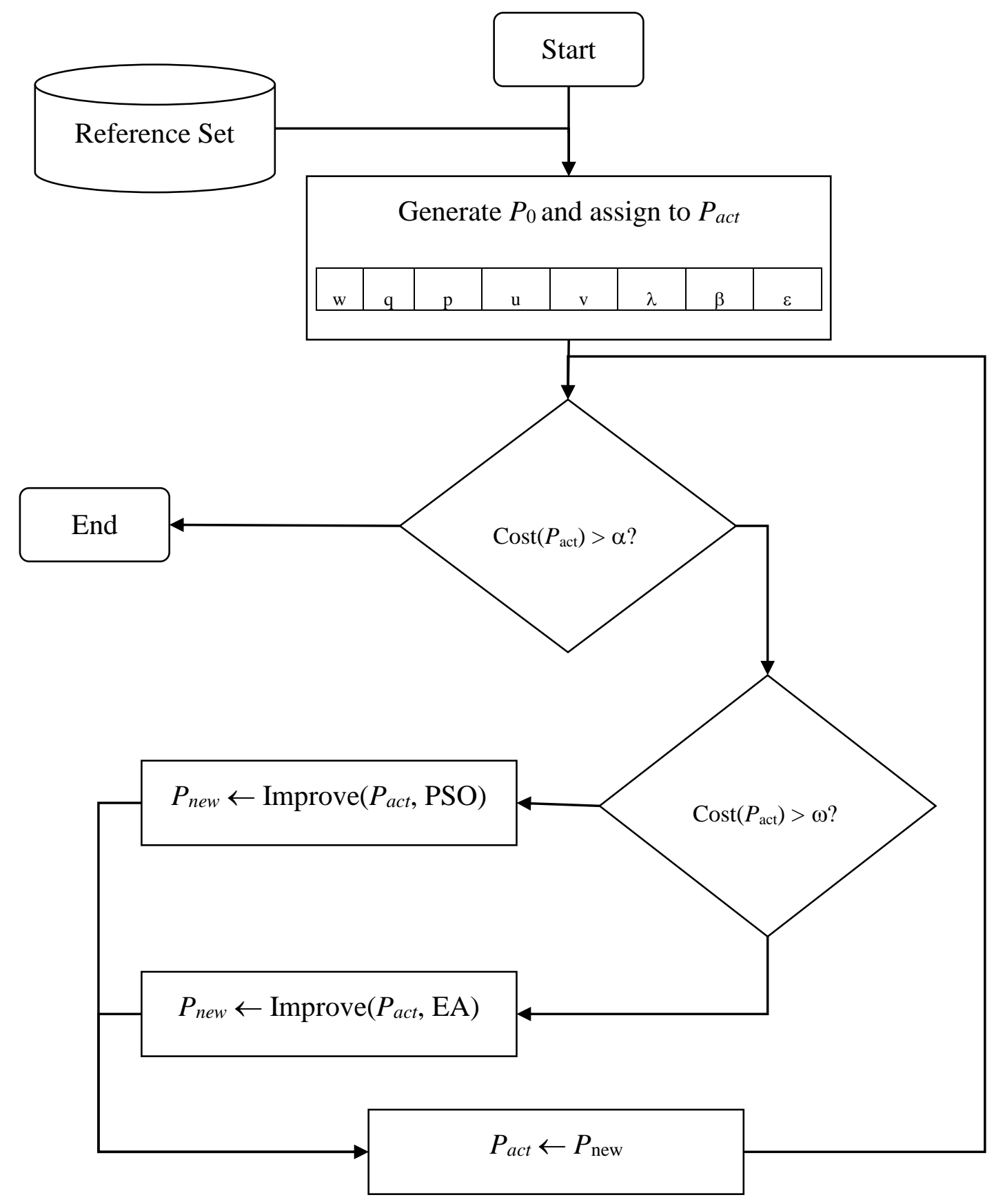

Figure 1. Generic architecture for hyperheuristics that elicit parameters of preference models.

The reference set is the stored battery of example previously provided by a decision maker, they can be preference relations stablished among pairs of alternatives, a whole set classified in distinct groups of interest, etc. The initial population is a collection of solutions that should be provided before the process begins, they can come from previous estimated values, random initialization, low level 
heuristics, etc., normally the individuals in the populations represents the set of parameters to be elicit, e.g. weights, indifference, preference, pre-veto, veto, and credibility thresholds used in ELECTRE and outranking methods. The performance model $\operatorname{Cost}\left(P_{i}\right)$ is an indicator of how would an actual population of solution is, usually it should be accompanied with thresholds that indicates when the population is good (e.g. any value greater than $\alpha$ is an indicator of a ill defined population), or when a different metaheuristic should be used (e.g. any value smaller than or equal to $\omega$ suggest the use of Particle Swarm Optimization [PSO], and any value greater recommend the use of Evolutionary Approaches [EA]). Finally, the improvement strategies are the metaheuristics considered for the evolution of a population (or transformation, depending on the approach used), here are denoted two commonly used in elicitation of parameters, PSO and EA.

\section{Conclusions}

The problem of eliciting parameters of a preference model can be tackle by means of a hyperheuristic. The method proposed is defined through an architecture that guides over the components that the hyperheuristic must have in order to improve the quality of the parameters estimated. To continue with the present work, it follows the implementation of the apprach, and the development of an experimental design in order to validate its performance.

\section{References}

Bechikh, S. (2013). Incorporating Decision Maker's Preference Information in Evolutionary Multiobjective Optimization, Diss. PhD thesis, High Institute of Management of Tunis, University of Tunis, Tunisia.

Branke, J., Salvatore, C., Greco, S., Slowiński, Zielniewicz, P. (2016). Using Choquet integral as preference model in interactive evolutionary multiobjective optimization. European Journal of Operational Research, 250:884-901.

Burke, E. K., Hyde, M. R., Kendall, G., Ochoa, G., Ozcan, E., \& Woodward, J. R. (2009). Exploring hyper-heuristic methodologies with genetic programming. In Computational intelligence (pp. 177201). Springer Berlin Heidelberg.

Burke, E. K., Hyde, M., Kendall, G., Ochoa, G., Özcan, E., \& Woodward, J. R. (2010). A classification of hyper-heuristic approaches. In Handbook of metaheuristics (pp. 449-468). Springer US.

Coello, C.A. (1999). A comprehensive survey of evolutionary-based multiobjective optimization techniques. Knowledge and Information Systems, an International Journal, 1(3):269-308. DOI:10.1007/ BF03325101.

Coello, C. C. (2000). Handling preferences in evolutionary multiobjective optimization: A survey. In Evolutionary Computation, 2000. Proceedings of the 2000 Congress on (Vol. 1, pp. 30-37). IEEE.

Coello, C. (2017). Introducción a la computación evolutiva. Notas de Curso CINVESTAV-IPN.

Cruz-Reyes, L, Fernandez, E., Rangel-Valdez, N. (2017). A metaheuristic optimization-based indirect elicitation of preference parameters for solving many-objective problems. International Journal of Computational Intelligence Systems, 10(2017): 56 - 77.

Dias, L., Mousseau, V. (2006). Inferring electre's veto-related parameters from outranking examples. European Journal of Operational Research, 170:172-191.

Fernandez, E., Lopez, E., Lopez, F., and Coello Coello, C.A. (2011). Increasing selective pressure towards the best compromise in evolutionary multiobjective optimization: The extended NOSGA method. Information Science (IS). 181: 44 - 56. 
Ling-Ko, L., Hsu, D., Lee, W.S., Ong, S.C.W. (2010). Structured Parameter Elicitation. APPEARED IN: Proc. AAAI Conference on Artificial Intelligence, 2010. Available at https://www.aaai.org/ocs/index.php/AAAI/AAAI10/paper/view/1798. Date accessed: 1/Nov/2018.

Miller, G.A. (1956). The magical number seven, plus or minus two: some limits on our capacity for processing information. Psychological Review, 63(2):81-97, 1956.

Rangel-Valdez, N. Fernandez, E., Cruz Reyes, L., Gomez-Santillan, C., Hernández-López, R.I. (2015). Multiobjective Optimization Approach for Preference-Disaggregation Analysis Under Effects of Intensity. MICAI (2) 2015: 451-462.

Roy, B. (1991). The outranking approach and the foundations of electre methods. Theory and Decisions, 31(1):49-73. DOI:

Soubeiga, E. (2003). Development and application of hyperheuristics to personnel scheduling (Doctoral dissertation, University of Nottingham).

Zhu, Y. \& Luo, Y. (2016) Multi-objective optimisation and decision-making of space station logistics strategies. International Journal of Systems Science, 47(13):3132 - 3148. 